\title{
Assessment of A New Magnetic Device to Monitor Swallowing in Parkinson's Disease
}

\author{
Toshiyuki Yamamoto ${ }^{1^{*}}$, Haruhi Inoguchi ${ }^{2}$, Yuko Sano ${ }^{3}$, Akihiko Kandori ${ }^{3}$ and Miho Murata ${ }^{1}$ \\ ${ }^{1}$ Department of Neurology, National Center Hospital, National Center of Neurology and Psychiatry, Tokyo, Japan \\ ${ }^{2}$ Rehabilitation Medicine, National Center Hospital, National Center of Neurology and Psychiatry, Tokyo, Japan \\ ${ }^{3}$ Central Research Laboratory, Hitachi, Ltd., Tokyo, Japan
}

"Corresponding author: Toshiyuki Yamamoto, Department of Neurology, National Center Hospital, National Center of Neurology and Psychiatry, 4-1-1 Ogawahigashicho, Kodaira City, Tokyo 187-8551, Japan, Tel: +81-42-341-2711; Fax: +81-42-346-1705; E-mail: yamamoto@ncnp.go.jp

Received date: Oct 22, 2014, Accepted date: Feb 17, 2015, Published date: Feb 23, 2015

Copyright: (c) 2015 Yamamoto T, et al. This is an open-access article distributed under the terms of the Creative Commons Attribution License, which permits unrestricted use, distribution, and reproduction in any medium, provided the original author and source are credited.

\begin{abstract}
Objective: To evaluate a magnetic swallowing monitoring device to monitor swallowing in patients with Parkinson's disease and to compare these findings with those of videofluoroscopic examination of swallowing (VF).

Methods: Twenty-one patients with Parkinson's disease and 11 healthy controls were included in this study. The subjects were examined by VF using $10 \mathrm{ml}$ of liquid barium, with the magnetic swallowing monitoring device attached to the patient's neck. We compared the results obtained from the magnetic swallowing monitoring device with those of VF.
\end{abstract}

Results: Eight patients and all controls showed normal swallowing on VF. According to the magnetic swallowing monitoring device, patients without dysphagia had similar waveforms to those of healthy controls. It also revealed the following swallowing-related abnormalities in 13 patients: piecemeal deglutition, delayed swallowing onset, delayed relaxation of the cricopharyngeal muscle, hesitation to swallow, and aspiration.

Conclusions: Patients with Parkinson's disease often showed abnormalities in the sequence of movements during the swallowing reflex. The magnetic swallowing monitoring device could detect these abnormalities simply and non-invasively.

Keywords: Parkinson's disease; Deglutition; Dysphagia; Magnetic device; Swallowing monitoring device; Video fluoroscopic examination of swallowing; Piecemeal deglutition; Aspiration

\section{Background and Purpose}

Video fluoroscopic examination of swallowing (VF) studies has reported various abnormalities in patients with Parkinson's disease (PD) [1-3]. In particular, aspiration is an important risk factor of pneumonia and discontinuation of oral intake in PD [4]. Although patients with PD require a periodical evaluation of their swallowing function, the frequency of VF should be ideally reduced, because VF involves X-ray exposure.

The process of swallowing involves sequential movements of the larynx [5]. The hyoid bone, which is used to identify the larynx on VF, moves upward and forward during swallowing, but no correlation has been observed between the amplitudes of the hyoid's upward and forward displacement [6,7]. The magnetic swallowing monitoring device (MSMD) is a new percutaneous system used to evaluate swallowing [8]. This device can determine the timing of the laryngeal movement on the basis of the distance between two magnetic coils as a "distance waveform" and the voltage of swallowing sounds detected by a piezoelectric microphone as a "sound waveform" in real time. The purpose of this study was to evaluate swallowing in patients with PD using the MSMD.

\section{Subjects and Methods}

The subjects included 21 patients with PD [median age: 70 years, interquartile range (IQR) 65-76 years; 9 men and 14 women; Hoehn Yahr (H\&Y) stage II, $\mathrm{n}=3$; stage III, $\mathrm{n}=13$; stage IV, $\mathrm{n}=4$; stage $\mathrm{V}, \mathrm{n}$ $=1]$ and 11 healthy controls (mean age: 23 years, IQR 29-45; 6 men and 5 women). Not all the subjects were aware of dysphagia and ingested their meals orally. The patients were selected from amongst patients with PD who were admitted to our hospital for evaluation or treatment of Parkinsonism. All patients were diagnosed with clinically definite PD [9] and were treated with L-DOPA. Cerebral infarction, arteriosclerotic parkinsonism, and other neurodegenerative diseases were excluded on the basis of cranial magnetic resonance imaging findings. 123I-metaiodobenzylguanidine (MIBG) scanning was also performed, and it was confirmed that the heart/mediastinum ratio of MIBG uptake declined to $\leq 1.6$ in the delayed phase [10].

An oscillation coil and a detection coil were built into the MSMD (Figure 1) [8]. The oscillation coil produced a magnetic field with a frequency of $20 \mathrm{kHz}$, and the inductive voltage in the detection coil was detected. The voltage was demodulated and passed through a lowpass filter (cut-off frequency $30 \mathrm{~Hz}$ ). The voltage was converted to a distance between the two coils by using calibration data. The piezoelectric microphone in the MSMD detected the swallowing sounds. The sound voltage detected by the microphone was rectified and passed through a low-pass filter (cut-off frequency $30 \mathrm{~Hz}$ ) to detect the envelope of the sound waveform. These two voltages (coil 
and sound voltages) were converted to digital data by an analog/digital converter with a sampling frequency of $100 \mathrm{~Hz}$ in the MSMD.
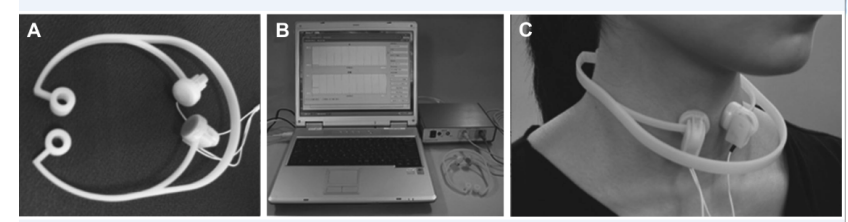

Figure 1: The magnetic swallowing monitoring device system. This system consists of three parts: the holder unit with the built-in coils and microphone (A), detection circuit unit, and personal computer to control and record the signals (B). The sensors of the holder unit can be attached to the front of the neck $(\mathrm{C})$.

VF was used to measure swallowing. Subjects were seated with the MSMD attached to the skin above the thyroid cartilage, and fluoroscopy was performed from the side. The position of the MSMD was determined by palpation of the thyroid cartilage. An investigator injected $10 \mathrm{ml}$ of a two-fold dilution of $110 \mathrm{w} / \mathrm{v} \%$ liquid barium into the subject's oral cavity; subsequently, the patient started swallowing in response to a signal. Patients who experienced wearing-off were tested during the "on" state. The fluorographic results were recorded on a DVD at 30 frames/s, and in all the clips, a marker was semiautomatically placed on the hyoid bone by using two-dimensional motion analysis software to evaluate the motion of the hyoid bone during swallowing. All subjects were provided with a written explanation of the VF procedure and of the use of MSMD during VF. Each subject provided written informed consent before beginning this study. This study was approved by the ethics committee of our institution.

The waveforms of the MSMD were sampled at $100 \mathrm{~Hz}$ and were synchronized with the fluorographic results on a personal computer.

The waveform of MDMS for the first swallowing was analyzed. A single assessor evaluated and compared all the results; another assessor re-evaluated the results of the healthy controls. The timing of each peak of the waveforms in the MSMD was compared using the Mann Whitney $\mathrm{U}$ test for normal swallowing and abnormal swallowing. $\mathrm{p}<$ 0.05 was considered significant. IBM SPSS $^{\star}$ (Ver.22) statistical software was used for these analyses.

\section{Results}

1) Normal swallowing dynamics: Nine of 21 patients and all controls showed normal swallowing on VF. In normal swallowing, the distance waveform in the MSMD detected five well-characterized peaks (P1, N2, P3, N4, and P5) (Figure 2A). Because a few peaks in the sound waveforms appeared for each subject, the highest acoustic peak was detected. To compare these findings with those of VF, the MSMD produced the distance waveform by the following mechanism. 1) The thyroid cartilage passed between the two coils of the MSMD with laryngeal elevation, and the distance between the coils narrowed (P1 to $\mathrm{N} 2$ ); 2) the coils were pushed out by the forward displacement of the larynx, and the distance between the coils was widened (N2 to P3); and 3) the thyroid cartilage moved backward (P3 to N4) and downward (N4 to P5), and the coils returned to their starting position (Figure 3). It is thought that the swallowing sounds are generated due to several factors [11]. On VF, liquid barium passed through the upper esophageal sphincter when the acoustic peak occurred between N2 and P5.

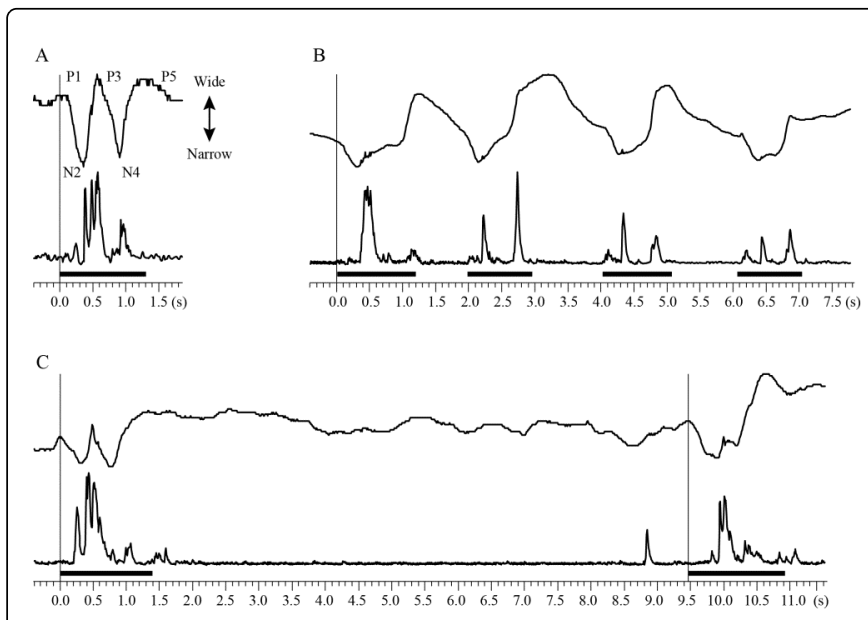

Figure 2: The swallowing waveform with the magnetic swallowing monitoring device. The upper waveform represents the distance waveform, and the lower waveform represents the sound waveform. The vertical line indicates the start of the swallowing. The solid lines above the temporal axis indicate the duration of swallowing. (A) Normal swallowing in a 48-year-old healthy man, (B) piecemeal deglutition in a 65-year-old woman with Parkinson's disease (PD), and (C) double swallowing in an 80-year-old woman with PD.

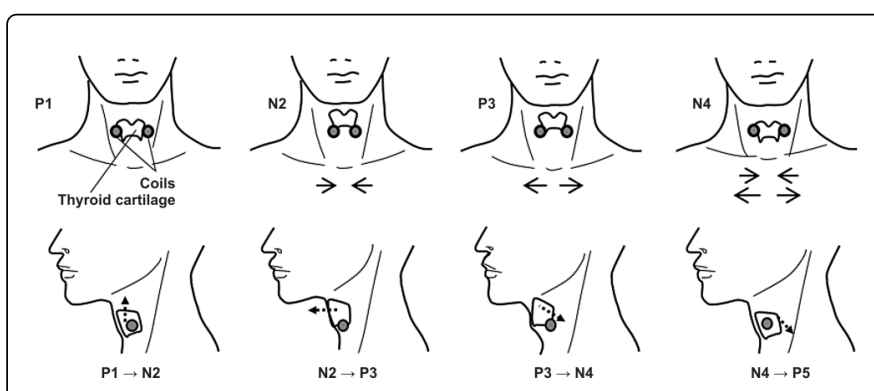

Figure 3: The mechanism of the waveform. The thyroid cartilage passes between the two coils during laryngeal elevation, and the distance between the coils decreases (P1 to N2). Then, the coils are pushed out by the anterior movement of the larynx (N2 to P3). Finally, the thyroid cartilage moves posteroinferiorly (P3 to N4), and the thyroid cartilage moves downward, returning to its starting position (N4 to P5). The upper solid arrow represents the distance between the coils, and the lower broken arrow represents the movements of the thyroid cartilage.

2) Abnormal swallowing dynamics: Twelve of 21 patients showed abnormal swallowing on VF.

a) Piecemeal deglutition was shown in four patients. VF showed that a small amount of liquid had flowed from the oral cavity to the pharynx, and a residue was observed in the oral cavity after swallowing. These patients had consumed the $10 \mathrm{ml}$ of liquid in several separate swallowing episodes. Patient 1 was a 65 -year-old woman with PD in H\&Y stage III. Swallowing reflexes were repeated four times in approximately $7.0 \mathrm{~s}$ during the consumption of $10 \mathrm{ml}$ of liquid barium 
(Figure 2B). The $\mathrm{P} 3$ peaks were small, and the $\mathrm{N} 4$ and $\mathrm{P} 5$ peaks were unclear in the distance waveform, but all swallowing reflexes showed a peak in the sound waveform.

b) Double swallowing was shown in five patients. After the first swallow, a residue was observed in the hypopharynx. A delayed second swallowing reflex was spontaneously evoked. Patient 2 was an 80 -yearold woman with PD in H\&Y stage IV. A second swallowing waveform appeared approximately $9.5 \mathrm{~s}$ after the appearance of the waveform for the first swallowing reflex (Figure 2C). Three healthy subjects had also swallowed the liquid in two swallows, but the interval between the first and second swallowing episodes was less than $5.0 \mathrm{~s}$.

c) Interruption of swallowing was shown in a patient. Patient 3 was a 72-year-old woman with PD in H\&Y stage III. After the start of the swallowing reflex, the interval from P1 to P3 was approximately $2.2 \mathrm{~s}$ (Figure 4A). The acoustic peak occurred between P3 and P5. After the liquid flowed into the pharynx, the swallowing reflex started, and the hyoid bone began to elevate. The hyoid bone, however, did not promptly move anteriorly, and relaxation of the cricopharyngeal muscle was also delayed. Finally, the liquid passed through the upper esophageal sphincter without aspiration on VF (Figure 4B).
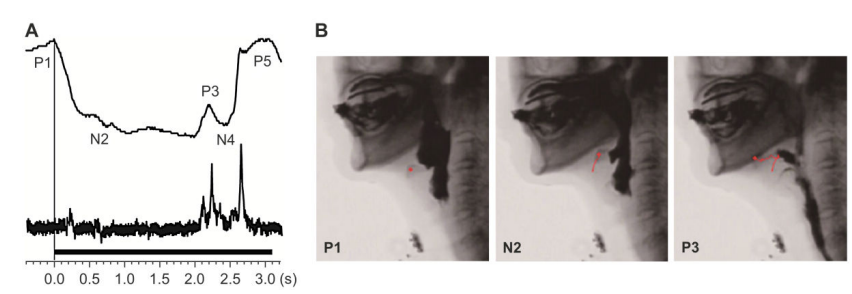

Figure 4: Interruption of swallowing in a 72-year-old woman with Parkinson's disease. (A) The swallowing waveform shown by the magnetic swallowing monitoring device. After the start of swallowing, the interval between P1 and P3 was approximately 2.2 s. The sound waveform nearly agreed with P3. The upper waveform represents the distance waveform, and the lower waveform represents the sound waveform. (B) On videofluorography, after the swallowing reflex had started (P1), the movement of the hyoid bone (red line) stopped with the larynx still elevated (N2). The hyoid bone then moved anteriorly, and the cricopharyngeal muscle opened. The liquid passed through the pharynx (P3).

d) Aspiration with hesitation was shown in two patients. On VF, after liquid had flowed from the oral cavity to the pharynx, laryngeal elevation did not start promptly and liquid was aspirated. Patient 4 was a 61-year-old man with PD in H\&Y stage III. At first, the distance waveform in the MSMD detected a movement in the larynx, but no accompanying acoustic peak was detected (Figure 5A, broken line). A shelving acoustic peak wave appeared with a time lag. Then, both the P3 peaks and acoustic peak appeared at $2.0 \mathrm{~s}$. Swallowing reflexes appeared as three events. Both the distance waveform and the sound waveform were disturbed. VF showed that laryngeal elevation was aborted without forward displacement, and the liquid had flowed into the larynx. When the liquid entered the larynx, barium bubbles were formed due to breathing. Then, three swallowing episodes were recorded. Finally, this patient aspirated the liquid and choked (Figure $5 B)$.

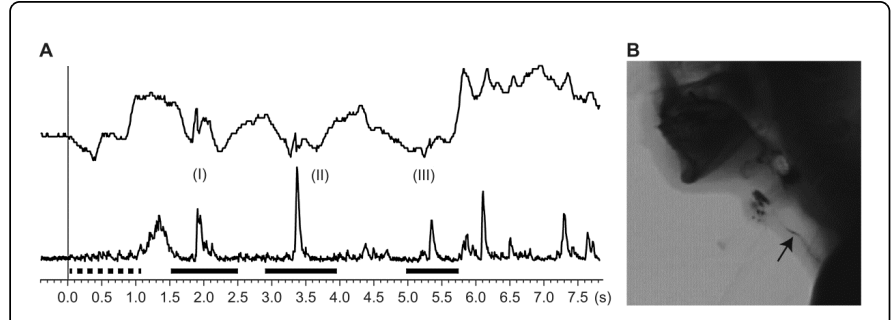

Figure 5: Hesitation to swallow and aspiration in a 61-year-old man with Parkinson's disease. (A) The swallowing waveform with the magnetic swallowing monitoring device. At first, movement of the larynx was observed, but no accompanying sound waveform peak was observed (the broken line above the temporal axis). A sound wave appeared with a time lag. A swallowing waveform appeared with an accompanying sound waveform (I). Swallowing then occurred twice (II, III). Afterward, the sound waveform was disordered. The upper waveform represents the distance waveform, and the lower waveform represents the sound waveform. (B) On videofluorography, this patient was found to have aspirated the liquid (arrow).

3) Timing analysis (Figure 6): We set the timing of P1 as a starting point. Compared to the normal swallowing dynamics, the abnormal swallowing dynamics exhibited significantly early timing of N2, P3, N4 and $\mathrm{P} 5$, respectively $(\mathrm{p}=0.02,=0.01,=0.01,<0.01)$. The outlier in the abnormal swallowing is Patient 3. The median timing of the acoustic peak appeared between P3 and N4. There was no significant difference in the timing of the acoustic peak between the two groups.

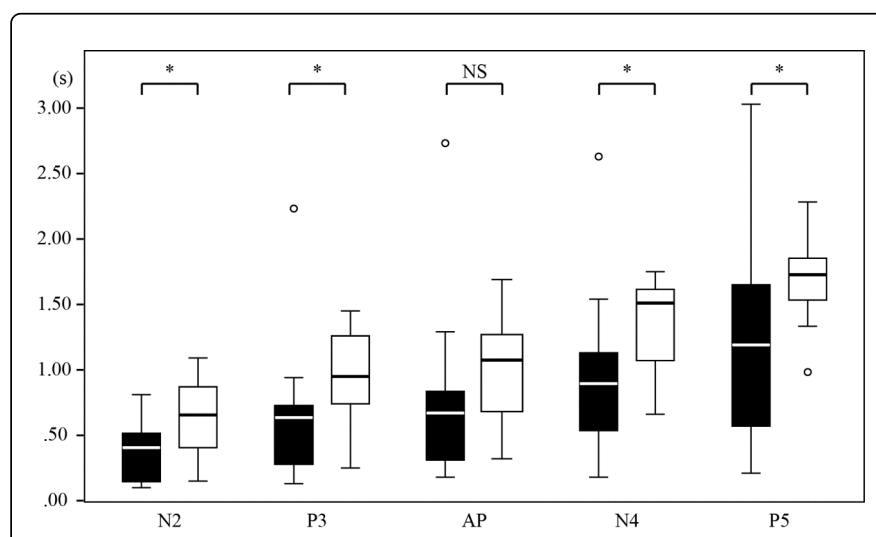

Figure 6: Box-and-whisker plot of the timing of the peaks of the waveforms in the MSMD. Compared to the normal swallowing dynamics, the abnormal swallowing dynamics exhibits significantly early timing of N2, P3, N4 and P5, respectively. AP, acoustic peak; Black bar, abnormal swallowing; white bar, normal swallowing; *, significant difference; NS, no significant difference.

\section{Discussion}

The dysphagia in PD is caused by a number of factors. In this study, we evaluated swallowing in patients with PD using the MSMD. The MSMD could evaluate not only the frequency and onset of swallowing reflex, but also abnormalities in the timing of swallowing processes in PD. 
Page 4 of 5

Dysfunction in transferring food from the mouth to the pharynx may be caused by lingual bradykinesia [12-14]. We believe that PD patients who showed piecemeal deglutition with MSMD had lingual bradykinesia.

It has been reported that the density of $\alpha$-synuclein-positive lesions in the pharyngeal sensory nerves is significantly greater in PD patients with dysphagia than in those without dysphagia, and these abnormalities may decrease pharyngeal sensation [15]. PD patients with decreased pharyngeal sensation could show delayed swallowing of residue in the pharynx with MSMD.

One characteristic of the swallowing abnormalities in PD is the disruption of the normal sequence in the pharyngeal phase of swallowing [16]. Dysfunction of the central pattern generators of swallowing in PD may be attributed to a deficit in the inhibition of the pedunculopontine tegmental nucleus $[17,18]$. In patient 3 , a break was observed in the swallowing reflex even though liquid had flowed into the pharynx. Moreover, patient 3 experienced delayed relaxation of the cricopharyngeal muscle. This type of dysphagia would be caused by dysfunction of the central pattern generators of swallowing. The MSMD was useful in evaluating the abnormalities of reflex-controlled sequential swallowing.

Once the liquid passes through the fauces, it can flow into the pharynx with the help of gravity, without the swallowing reflex [19]. In patients with aspiration, the liquid had flowed into the pharynx before the swallowing reflex occurred; in addition, these patients showed hesitation in swallowing and aspiration. Our findings suggest that the mismatch between the position of liquid and the timing of the pharyngeal process may be a cause of dysphagia in PD. Therefore, it might be important to evaluate both the movement of the larynx and the position of the liquid during swallowing in PD. The videofluorographic method and the MSMD system could complement each other in swallowing studies.

The thyroid cartilage moved faster in abnormal swallowing than in normal swallowing. Moreover, there was no significant difference in the timing of the acoustic peak between the two groups, which suggests that PD patients with abnormal swallowing swallow in a short time, resulting in incomplete swallowing.

Recently, it has been reported that targeted training of sensorimotor actions with swallowing would improve swallowing in PD [20]. It is an effective treatment that helps PD patients with dysphagia recognize the difference between normal and abnormal swallowing [21]. The MSMD may be useful in targeted exercise therapy for swallowing in PD, because patients can use this device repetitively and monitor their swallowing in real time.

This study has several limitations. First, the small sample size could limit the reliability of the study. Second, it is possible that the waveforms of MSMD will change depending on the consistency and volume of the food used for examination.

To conclude, the MSMD could detect both normal and abnormal swallowing in PD simply and non-invasively. When using this system during VF, the dysfunction of the central pattern generators of swallowing in PD could be diagnosed easily. In the future, we plan to investigate more patients with PD and use the MSMD for targeted exercise therapy for swallowing in PD.

\section{Acknowledgements}

The MSMD was provided by Advanced Research Laboratory, Hitachi, Ltd., Tokyo, Japan.

\section{References}

1. Bushmann M, Dobmeyer SM, Leeker L, Perlmutter JS (1989) Swallowing abnormalities and their response to treatment in Parkinson's disease. Neurology 39: 1309-1314.

2. Ali GN, Wallace KL, Schwartz R, DeCarle DJ, Zagami AS, et al. (1996) Mechanisms of oral-pharyngeal dysphagia in patients with Parkinson's disease. Gastroenterology 110: 383-392.

3. Umemoto G, Tsuboi Y, Kitashima A, Furuya H, Kikuta T (2011) Impaired food transportation in Parkinson's disease related to lingual bradykinesia. Dysphagia 26: 250-255.

4. Yamamoto T, Kobayashi Y, Murata M (2010) Risk of pneumonia onset and discontinuation of oral intake following videofluorography in patients with Lewy body disease. Parkinsonism Relat Disord 16: 503-506.

5. Logemann JA (1998) Evaluation and treatment of swallowing disorders. (2nd edn), Pro-Ed Publishers, Austin, Texas.

6. Ishida R, Palmer JB, Hiiemae KM (2002) Hyoid motion during swallowing: factors affecting forward and upward displacement. Dysphagia 17: 262-272.

7. Palmer JB, Tanaka E, Ensrud E (2000) Motions of the posterior pharyngeal wall in human swallowing: a quantitative videofluorographic study. Arch Phys Med Rehabil 81: 1520-1526.

8. Kandori A, Yamamoto T, Sano Y, Oonuma M, Miyashita T, et al. (2012) Simple magnetic swallowing detection system. IEEE Sensors Journal 12: 805-811.

9. Calne DB, Snow BJ, Lee C (1992) Criteria for diagnosing Parkinson's disease. Ann Neurol 32: S125-S127.

10. Yoshita M (1998) Differentiation of idiopathic Parkinson's disease from striatonigral degeneration and progressive supranuclear palsy using iodine-123 meta-iodobenzylguanidine myocardial scintigraphy. J Neurol Sci 155: 60-67.

11. Cichero JA, Murdoch BE (1998) The physiologic cause of swallowing sounds: answers from heart sounds and vocal tract acoustics. Dysphagia 13: 39-52.

12. Nagaya M, Kachi T, Yamada T, Igata A (1998) Videofluorographic study of swallowing in Parkinson's disease. Dysphagia. 13: 95-100.

13. Robbins JA, Logemann JA, Kirshner HS (1986) Swallowing and speech production in Parkinson's disease. Ann Neurol 19: 283-287.

14. Logemann J, Blonsky ER, Boshes B (1973) Lingual control in Parkinson's disease. Transactions of the American Neurological Association. 98: 276-278.

15. Mu L, Sobotka S, Chen J, Su H, Sanders I, et al. (2013) Parkinson disease affects peripheral sensory nerves in the pharynx. J Neuropathol Exp Neurol 72: 614-623.

16. Hunter PC, Crameri J, Austin S, Woodward MC, Hughes AJ (1997) Response of parkinsonian swallowing dysfunction to dopaminergic stimulation. J Neurol Neurosurg Psychiatry 63: 579-583.

17. Alfonsi E, Versino M, Merlo IM, Pacchetti C, Martignoni E, et al. (2007) Electrophysiologic patterns of oral-pharyngeal swallowing in parkinsonian syndromes. Neurology 68: 583-589.

18. Ertekin C, Tarlaci S, Aydogdu I, Kiylioglu N, Yuceyar N, et al. (2002) Electrophysiological evaluation of pharyngeal phase of swallowing in patients with Parkinson's disease. Mov Disord 17: 942-949.

19. Saitoh E, Shibata S, Matsuo K, Baba M, Fujii W, et al. (2007) Chewing and food consistency: effects on bolus transport and swallow initiation. Dysphagia 22: 100-107.

20. Russell JA, Ciucci MR, Connor NP, Schallert T (2010) Targeted exercise therapy for voice and swallow in persons with Parkinson's disease. Brain Res 1341: 3-11. 
Citation: Yamamoto T, Inoguchi H, Sano Y, Kandori A, Murata M (2015) Assessment of A New Magnetic Device to Monitor Swallowing in Parkinson's Disease. J Neurol Neurophysiol 6: 267. doi:10.4172/2155-9562.1000267

Page 5 of 5

21. Manor Y, Mootanah R, Freud D, Giladi N, Cohen JT (2013) Videoassisted swallowing therapy for patients with Parkinson's disease. Parkinsonism \& Rel Disord 19: 207-211. 